INTERVIEW

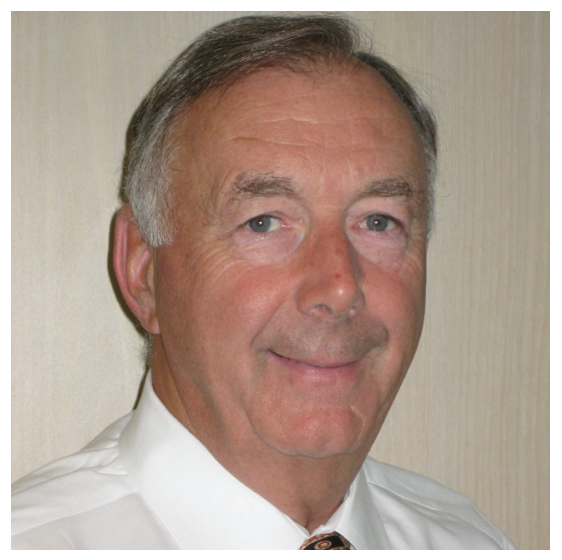

\title{
StemCells, Inc.: clinical trials of stem cell therapies for CNS disorders
}

\author{
Interview with Martin McGlynn
}

Martin McGlynn, President \& Chief Executive Officer of StemCells, Inc., talks to Regenerative Medicine about the company's ongoing clinical trials with their human neural stem cell treatment (HuCNS-SC ${ }^{\circledR}$ ).

Martin McGlynn joined StemCells, Inc. in January of 2001 as President and Chief Executive Officer, and was elected to its Board of Directors on February 6, 2001. Martin McGlynn has spent several decades as a senior executive in the life sciences industry in Europe, Canada and the USA. He began his career in Manufacturing Operations with Becton Dickinson, Ireland Ltd. then joined Abbott Labs in 1977 where he held positions as General Manager, Abbott Ireland Ltd, President and General Manager of Abbott Canada Ltd and Vice President of Abbott International Ltd. Martin McGlynn currently serves as a member of the Board of the Alliance for Regenerative Medicine and is co-Chairman of its Operations and Governance Committee.
Tell us a little about the company's approach to developing adult stem cell treatments.

StemCells, Inc. takes an approach that we call homologous use of stem cells, meaning we take cells from brain tissue and put them back into the brain; we take liver cells and put those back into the liver; and we put pancreas cells back into the pancreas. We do it this way because we are working with adult stem cells. Unlike an embryonic stem cell, which can give rise to every cell type in the body (pluripotent), adult stem cells are essentially hard-wired to their specific organ system. For example, the cell type we are working with for CNS indications, human neural stem cells $\left(\mathrm{HuCNS}-\mathrm{SC}^{\circledR}\right)$, are hard-wired to

\section{In all the transplants we have done (in thousands of animals and 13 patients), we have seen no evidence of tumorigenic
activity...}

become the three main cellular actors of the CNS - neurons, astrocytes and oligodendrocytes - while maintaining a pool of stem cells for lifelong repair and replacement of these specialized cells of the CNS.

The cells we use are unmodified - we do not genetically alter them. They are expandable to commercial scale in cell banks, so it is a business model that is focused on product development as opposed to a patient-by-patient service business. We can grow billions of cells in master cell banks and cryopreserve them ready to make patient products for transplant: these really are stem cells in a bottle. These cells are hard-wired to become only cells of the CNS, they can be directly transplanted as such and are 
believed to be nontumorigenic - they do not give rise to tumors (in vitro or in vivo). In all the transplants we have done (in thousands of animals and 13 patients), we have seen no evidence of tumorigenic activity, so the US FDA is increasingly comfortable with the safety profile of these cells being transplanted into humans.

So what happens when you put these cells into a living host? They engraft, they migrate and then they differentiate into neurons, astrocytes or oligodendrocytes in a site-specific way. These cells take their cues from the host microenvironment and, depending upon the host organ, whether it is in the brain or spinal cord, they will predominately become one or another of the cells, and start carrying out the cells' assigned function. This opens up a very broad spectrum for applications in the clinic. It is an amazing cell, it does amazing things when transplanted into animals, and we are now in the business of replicating what we've seen in human patients.

What made StemCells, Inc. choose to explore an allogeneic rather than autologous approach?

Put simply, allogeneic cells can be expanded into millions of cells, which you can then use to treat thousands of patients, whereas an autologous approach involves taking patient tissue, processing it in some way and then putting it back into the patient. It is more like a service or medical procedure than a product business model. In a liver transplant you transplant one patient from one donor. In the case of allogeneic therapies, it could be thousands of patients from one donor.

The benefit of the autologous approach is that the patient recognizes the cells as self. So unless there is something done to the cells to alter them outside the body, the likelihood that they will be rejected when transplanted back into the donor is very low. With the allogeneic approach we use, where you take donor tissue and transplant it into a host, the host immune system will recognize the cells from donor tissue as foreign. So the immune system will immediately go about setting up defences, and in time would reject the donor cells. Therefore, when you are developing allogeneic transplant methodologies, you also have to develop immunosuppressant regimens. In the CNS, the immunosuppression is temporary: we stop the immunosuppressant regimen after 9 months because by that time it seems the cells have integrated into the body and the host has accommodated them. Importantly, we do know from trials we have done, the Batten disease study for example, that not only do the cells survive with the immunosuppressant regimen and do so for long periods of time, but the cells survive way beyond cessation of the immunosuppression.

\section{Could you give us an overview of the clinical trials that are underway at the company?}

We are the only company worldwide to my knowledge that has clinical trials underway in all three regions of the CNS - the brain, the spinal cord and the eye. All of these trials are using the same cells - HuCNS-SCs. The first clinical trial we did was in a fatal lysosomal storage disease, Batten disease. It was the first ever human neural stem cell transplantation. We transplanted over a billion cells into a human brain, with the aim of using the cells to deliver enzymes that were missing in the brains of these patients. We completed that Phase I study in January 2009. The results from the Phase I study were very promising. This is a progressive and invariably fatal disease, and the children being treated were at an advanced stage. Of the six transplanted patients, three of them are still alive today two patients in the study are, more than 5 years after treatment. It is hard to go beyond that observation because it was not a controlled clinical study, but my $\mathbf{6}$ The benefit of the autologous approach is that the patient recognizes the cells as self. 99 
opinion is that you cannot rule out the possibility that the cells have helped these surviving children. A Phase II trial was approved by the FDA, but could not be completed due to insufficient enrolment.

The second study we launched was in Pelizaeus-Merzbacher disease (PMD), a fatal hypomyelination disorder. These patients have no myelin orvery little myelin and are incapable of generating myelin. When the brain's myelin is damaged or insufficient (hypomyelinated), the axons die and the patient dies in childhood. Here we are using the same neuronal stem cells to myelinate the host axons that are hypomyelinated. We completed the PMD trial in February of this year. We have announced top-line results and the detailed results of that study are under review in a peer-reviewed journal. All four patients undergoing transplantation in that study were shown to have no myelin on their nerve axons at baseline. Three of the four patients presented small but measureable improvement in neurological markers, while the fourth patient was clinically stable. This was a remarkable observation. Not only did the cells engraft and survive, but they are biologically active, and they appear to have conferred a clinical benefit to these patients.

The next trial we initiated was in spinal cord injury, a Phase I/II trial being carried out in Switzerland and authorized by Swissmedic. Our study design means that we start off with the worst of the worst-affected patients, the ASIA A population who have no sensory or motor function below the level of injury. We have dosed all three ASIA-A patients in the first cohort, and have started enrolling the ASIA B's, who have incomplete injury. We enroll patients 3-12 months postinjury. This makes it very different to the Geron study, which was enrolling patients within a couple of weeks of spinal cord injury. Our view is that if you put cells in too early they may not survive in what is a very hostile inflammatory environment, and if there is an effect, good or bad, it is hard to know whether it is caused by the cells or by progression of the injury. We reported safety data in May of this year, showing a very good safety profile, and an exciting development is that two of the patients are reporting improved sensation to light touch below the site of injury. We plan to be in London, UK, in September to present data on the 6-month evaluation of these patients, which we are all looking forward to.

\section{We are the only company worldwide to my knowledge that has clinical trials underway in all three regions of the CNS - the brain, the spinal cord and the eye. $\boldsymbol{M}$}

The fourth trial is in age-related macular degeneration. We had a Phase I/II study authorized by the FDA in January 2012, and initiated the study in June 2012 at the Retina Foundation of the Southwest's Anderson Vision Research Center in TX, USA. In a rat model of macular degeneration, we have shown that neural stem cells can do some remarkable things, and effectively preserve vision, so we are very excited to be moving into clinical trials for AMD.

\section{What new clinical trials do you hope to initiate over the next few years?}

We recently got a US\$20 million award from the California Institute for Regenerative Medicine to initiate INDenabling activities for a trial in cervical spinal cord injuries, which occur a little further up the spine, closer to the neck. That is where the majority of spinal cord injuries occur. Our game plan is to initiate clinical trials within 4 years, by which time we should have data from the thoracic spinal cord injury clinical trial.

In preclinical development is a very important program in Alzheimer's disease, for which we have again applied for funding of up to US $\$ 20$ million for IND-enabling activities. What we have shown is that HuCNS-SCs are able to enhance memory in animal models of Alzheimer's disease. And they do so independent of eradicting plaques and tangles. It is a very intriguing and controversial observation, especially because there has been little success clinically using strategies targeting plaques.

The Batten and PMD trials enrolled children with genetic brain disorders. Are there any special challenges in conducting clinical trials in children?

There are elevated regulatory and ethical considerations when you conduct any clinical trials in children. There is a heightened sense of scrutiny with regards to the risk-reward equation. Is there a body of evidence that suggests that it is reasonably safe to put these cells into children? And secondly, is there a likelihood that the children might benefit from the procedure? In the case of adults you certainly have to be able to demonstrate that it is reasonably safe, but the expectation in terms of the clinical benefit for the subject is not as high.

The Batten disease program did not progress to a Phase II trial due to a lack of enrolment. Is enrolment likely to be problem for the PMD program?

In the case of the first study, we put the cells into the worst-affected children - most of their neurons had already died so there was very little left to protect with our cells. So the purpose of the second study was to go into patients who had most of their neurons intact. That means we had to find them much earlier. What we found after 6 months of trying is that we could not identify a single patient who met the study criteria. The simple reason is that it takes our healthcare system too long to come up with the correct diagnoses for these children. Children 
with Batten disease for all intents and purposes look very healthy - there are no immediate tell-tale signs. Over time, the parents start to notice their children are not hitting developmental milestones and start to get seizures. But often the children are initially misdiagnosed and treated with seizure meds. It is only after the seizure meds do not control the disease that eventually somebody orders the diagnostic tests. It takes more than 2 years on average, by which time pathology is too severe and they have lost most of their neurons. So we were forced to shelve the program, not because we were not confident about the technology and the ability to help these kids, but until such a time as our healthcare system has addressed the problem and is able to diagnose much earlier. We are definitely open to re-intiating the trial if that situation does come about. We are in a state of watchful waiting.

PMD is another rare disease but in children with the most severe form, connatal PMD, it is obvious from infancy that there is something wrong so these children are diagnosed very early. We even had a 14-month-old baby in our trial. So we should not have the same problem we had with Batten disease of identifying early-stage patients.

\section{How do you think the field of stem cell therapy will develop over the next $5-10$ years?}

The field is maturing rapidly. The rate of progress in opening up clinical trials and advancing towards the clinic has accelerated exponentially. In the case of StemCells, Inc., over the next 3-4 years we will have human clinical data and results of clinical trials from the brain, the spinal cord and the eye.

However, it is not an easy path to the clinic. I think the two biggest challenges facing the industry are being able to separate the good science from the bad and to sustain funding.
It is going to become more and more difficult to discern what is important and what is not, to separate the wheat from the chaff. It is always challenging for patients and their families, as well as investors, to cut through all the hype and figure out what is real. I can only speak for StemCells, Inc. and say we have done all the heavy lifting when it comes to the scientific premise, the preclinical data, and the rigor and design of our clinical trials.

In terms of funding, there is a big question mark over who is going to fund companies engaged in translation through the 'valley of death' between proof of principle and the clinic. The venture capital community appears disinterested in the stem cell space and the public investor community is skittish, sceptical and confused. Ultimately, until such time as we have clinical data proving efficacy, it is going to be a struggle. Thankfully, in California, the California Institute for Regenerative Medicine is like the US cavalry as far as we are concerned: we have been waiting for them to come over the hill! They are certainly going to be a game changer in terms of helping companies like ours make it through the valley of death and come out the other side with human clinical data.

Financial \& competing interests disclosure

M McGlynn is a director and employee of StemCells, Inc. ("Company"), the sponsor of the preclinical and clinical studies described in the manuscript. The Company has previously disclosed, in SEC filings, press releases and other corporate communications, the clinical and preclinical information discussed in the manuscript. The author has no other relevant affiliations or financial involvement with any organization or entity with a financial interest in or financial conflict with the subject matter or materials discussed in the manuscript apart from those disclosed.

No writing assistance was utilized in the production of this manuscript.
66 challenges facing the industry are being able to separate the good science from the bad and to sustain funding... 99 